\title{
Functional Reorganizations of Brain Network in Prelingually Deaf Adolescents
}

\author{
Wenjing Li, ${ }^{1,2,3}$ Jianhong Li, ${ }^{4}$ Jieqiong Wang, ${ }^{2}$ Peng Zhou, ${ }^{2}$ Zhenchang Wang, \\ Junfang Xian, ${ }^{4}$ and Huiguang $\mathrm{He}^{2}$ \\ ${ }^{1}$ College of Electronic Information and Control Engineering, Beijing University of Technology, Beijing 100124, China \\ ${ }^{2}$ State Key Laboratory of Management and Control for Complex Systems, Institute of Automation, \\ Chinese Academy of Sciences, Beijing 100190, China \\ ${ }^{3}$ Beijing Key Laboratory of Computational Intelligence and Intelligent System, Beijing 100124, China \\ ${ }^{4}$ Department of Radiology, Beijing Tongren Hospital, Capital Medical University, Beijing 100730, China \\ ${ }^{5}$ Department of Radiology, Beijing Friendship Hospital, Capital Medical University, Beijing 100050, China
}

Correspondence should be addressed to Junfang Xian; cjr.xianjunfang@vip.163.com and Huiguang He; huiguang.he@ia.ac.cn

Received 5 June 2015; Accepted 22 July 2015

Academic Editor: Feng Shi

Copyright (C) 2016 Wenjing Li et al. This is an open access article distributed under the Creative Commons Attribution License, which permits unrestricted use, distribution, and reproduction in any medium, provided the original work is properly cited.

Previous neuroimaging studies suggested structural or functional brain reorganizations occurred in prelingually deaf subjects. However, little is known about the reorganizations of brain network architectures in prelingually deaf adolescents. The present study aims to investigate alterations of whole-brain functional network using resting-state fMRI and graph theory analysis. We recruited 16 prelingually deaf adolescents (10 18 years) and 16 normal controls matched in age and gender. Brain networks were constructed from mean time courses of 90 regions. Widely distributed network was observed in deaf subjects, with increased connectivity between the limbic system and regions involved in visual and language processing, suggesting reinforcement of the processing for the visual and verbal information in deaf adolescents. Decreased connectivity was detected between the visual regions and language regions possibly due to inferior reading or speaking skills in deaf subjects. Using graph theory analysis, we demonstrated smallworldness property did not change in prelingually deaf adolescents relative to normal controls. However, compared with healthy adolescents, eight regions involved in visual, language, and auditory processing were identified as hubs only present in prelingually deaf adolescents. These findings revealed reorganization of brain functional networks occurred in prelingually deaf adolescents to adapt to deficient auditory input.

\section{Introduction}

Prelingual deafness is the hearing loss that occurs at birth or before the onset of speech. Due to the deprivation of auditory inputs, brain plasticity has been reported by numerous neuroimaging studies. "Cross-modal plasticity" has been suggested in deaf subjects, which is represented by the phenomenon that the auditory cortex can be activated when deaf subjects perform various tasks, such as speech $[1,2]$ and visual tasks $[3,4]$. Many morphological studies did not find structural changes in the primary auditory cortex [5-8], indicating the atrophy of the auditory cortex due to hearing loss could be compensated by the use of this cortex for other stimuli. However, brain regions involved in visual and speech processing have been found to change in deaf subjects $[7,8]$, indicating that the sensory systems for vision and speech might participate in the tasks which are supposed to activate the auditory system in healthy controls. Therefore, we speculated that the cooperative manner for different brain regions would be altered in prelingually deaf subjects when dealing with a complex task.

The human brain is a highly complex system with synchronized neural activity from different brain regions. The concept of "connectome" was first proposed by Sporns et al. in 2005 [9], which represents the human brain as an interconnected network. Functional brain network refers to a pattern of statistical dependencies between distinct brain regions. The network architecture identified by the analysis 
of functional connectivity could be an effective pattern to present the cooperative manner for brain regions, and it reflects the potential anatomical connections between brain regions as well. To further quantitatively measure the brain network, network topological properties could be evaluated at both global and regional levels using graph theory, which becomes a promising tool for analyzing brain networks in recent years [10-21]. Small-worldness, a concept that originated from social network, quantifies the effectiveness of information transferring within networks and has been successfully used to characterize brain networks [14-16]. Besides, nodal topological parameters based on graph theory analysis are used to illustrate the properties for nodes which are defined as brain regions, identifying the role of specific regions in transferring information. It has been demonstrated that these measures of brain network are sensitive to aging $[17,18]$ as well as various neuropsychiatric diseases, such as schizophrenia [16, 19] and Alzheimer's disease [20, 21]. Therefore, investigation of brain network properties provides a new insight into brain reorganization and is critical to understand the working mechanism of brains with hearing loss.

Recently, a few studies have emerged to investigate the structural or functional connectivity between brain regions in deaf subjects. Kim et al. [22] examined morphological brain network in deaf adults using tissue density on MRI and analyzed the network properties using graph theory and network filtration. They found altered morphological network in prelingually deaf adults compared to normal controls but not in postlingually deaf adults, concluding that auditory experience could affect the morphology of brain networks in deaf adults. Besides, Li et al. [23] employed resting-state fMRI to investigate the effect of deafness on the intra- and interregional synchronization of different parts of superior temporal sulcus and revealed that the intrinsic function of these different parts are distinctly impacted by deafness. However, these previous studies focused on alterations in grown adult brains of deaf subjects but not developing brain. Adolescence is an important transitional stage for brain development, in which brain's structure and function are in developmental changes to accommodate to external environment. Therefore, adolescents with prelingual deafness are supposed to go through a different pattern of brain reorganization to adapt to the silent world relative to normal adolescents, which is supported by one of our previous studies [24] using structural MRI to investigate grey matter connectivity within and between auditory, language, and visual systems in deaf adolescents. To the best of our knowledge, the whole-brain functional network and its properties have not been investigated in prelingually deaf adolescents yet.

We hypothesized the architectures or properties of brain network would change in prelingually deaf adolescents. In the present study, we used resting-state fMRI and graph theory analysis to investigate alterations of whole-brain functional networks in prelingually deaf adolescents. Functional brain network was constructed based on mean time series extracted from 90 brain regions. Functional connectivity patterns were studied in deaf and control groups, respectively, and compared between two groups. The global network
TABLE 1: Biographical information of two groups.

\begin{tabular}{lcc}
\hline & Normal controls & Deaf adolescents \\
\hline Number & 15 & 15 \\
Male/female & $8 / 7$ & $8 / 7$ \\
Mean age (years) & 14.81 & 14.32 \\
Standard deviation & 2.07 & 2.24 \\
(SD) age (years) & $10 \sim 18$ & $10 \sim 18$ \\
Age range (years) & & \\
\hline
\end{tabular}

property of small-worldness was evaluated in each group and group differences were investigated as well. Besides, nodal topological parameters were calculated and brain hubs were identified for deaf and control groups, respectively. Nodal properties were finally compared in all the hubs between prelingually deaf adolescents and normal controls.

\section{Materials and Methods}

2.1. Subjects. In this study, we recruited 16 prelingually deaf adolescents and 16 normal controls with matched age ( $p=$ 0.815 ) and gender (Chi-Squared $p=1.0$ ), which were the same as the data used in our previous studies $[7,8$, 24]. All prelingually deaf adolescents suffered from severe sensorineural hearing loss after the birth and had a mean pure tone audiometry average air conduction threshold greater than $90 \mathrm{~dB}$ of hearing loss for the better ear and no single frequency better than $45 \mathrm{~dB}$ of hearing loss between 500 and $4000 \mathrm{~Hz}$. All deaf subjects wore hearing aids and learnt Chinese sign language. Furthermore, all subjects had neither history of central nervous system disease nor significant head trauma. All subjects underwent an identical restingstate fMRI paradigm. The data from one prelingually deaf adolescent and one normal control were discarded due to excessive head movement during scanning. Therefore, 15 deaf subjects (age 14.32 \pm 2.24 years, $10 \sim 18$ years; 8 males) and 15 normal controls (age $14.81 \pm 2.07$ years, 10 18 years; 8 males) remained, and these two groups were still matched in age $(p=0.769)$ and gender (Chi-Squared $p=1.0)$. Detailed biographical information of all subjects is shown in Table 1. This project was approved by the Committee at Beijing Tongren Hospital, and all subjects and their parents gave written informed consent before inclusion.

2.2. Data Acquisition. Resting-state fMRI data were collected from all subjects on a 3-Tesla MR imaging scanner (GE Medical System, Milwaukee, WI, USA) with an eight-channel phased-array head coil. To acquire resting-state fMRI data, all subjects lay on their backs and were instructed explicitly to keep their eyes closed and not to think of anything in particular. Foam padding was used to limit head motion and reduce scanner noises. The scanning parameters were as follows: repetition time $(\mathrm{TR})=2000 \mathrm{~ms}$, echo time $(\mathrm{TE})=$ $30 \mathrm{~ms}$, field of view $(\mathrm{FOV})=24 \times 24 \mathrm{~cm}^{2}$, matrix size $=64 \times$ 64 , slice thickness $=5 \mathrm{~mm}$, and flip angle $=90^{\circ}$, yielding 28 axial slices with in-plane resolution of $3.75 \times 3.75 \mathrm{~cm}^{2}$. The fMRI scanning lasted $400 \mathrm{~s}$ and 200 volumes were obtained for each subject. 
Furthermore, high-resolution T1-weighted structural brain images were collected for structural reference using a 3D SPGR sequence $(\mathrm{TR}=9 \mathrm{~ms}, \mathrm{TE}=3.5 \mathrm{~ms}$, inversion time $(\mathrm{TI})=450 \mathrm{~ms}, \mathrm{FOV}=24 \times 24 \mathrm{~cm}^{2}$, matrix size $=256 \times 256$, slice thickness $=1 \mathrm{~mm}$, and flip angle $=13^{\circ}$, yielding 196 sagittal slices with in-plane resolution of $0.9375 \times 0.9375 \mathrm{~cm}^{2}$ ).

2.3. Data Preprocessing. The preprocessing steps were performed using the Statistical Parametric Mapping (SPM8, http://www.fil.ion.ucl.ac.uk/spm, Wellcome Department of Cognitive Neurology, London, UK, 2008) on a Matlab 7.9 platform (MathWorks, Natick, MA, USA). The first 10 volumes of the resting-state fMRI data were discarded because of the initial transient effects and the adaption of the subjects to the environment. The remaining 190 time points were corrected for within-scan acquisition time differences between slices and realigned to the first image for head movement correction, resulting in 3 translational and 3 rotational parameters. The subjects with excessive head movement (translation $>2 \mathrm{~mm}$ and rotation degree $>3^{\circ}$ ) were thus excluded. Subsequently, the functional scans were spatially normalized to a standard space (Montreal Neurological Institute, MNI) using the normalization parameters from $\mathrm{T} 1$ image with high resolution to structural T1 template in MNI space and resampled to $3 \times 3 \times 3 \mathrm{~mm}^{3}$. The images were then smoothed with a $6 \mathrm{~mm}$ full width at half maximum (FWHM) isotropic Gaussian kernel. After that, the preprocessed images were linearly drifted and passed through a band-pass filter $(0.01-0.08 \mathrm{~Hz})$ to remove the effects of low frequency drift and physiological high frequency respiratory and cardiac noise. We regressed out six parameters of head movement as well as mean signals of the whole brain, white matter, and cerebral spinal fluid.

2.4. Network Construction. The functional images were parcellated into 90 brain regions (45 regions for each hemisphere) based on Automated Anatomical Labelling (AAL) template (see Table 2). To construct the whole-brain network, time courses were first extracted from all voxels within each ROI and averaged. Then, Pearson's correlation coefficient was calculated between pairs of regions throughout the whole brain, resulting in a $90 \times 90$ correlation matrix. Larger correlation coefficients indicate more synchronized time courses between the corresponding pairs of regions, implying stronger functional connectivity between these two regions.

2.5. Graph Theory Analysis. Brain network properties were analyzed using graph theory based on the correlation matrix, in which 90 ROIs and their connections were considered as nodes and edges, respectively. The correlation matrix was binarized by a given threshold, resulting in a sparse matrix. We adjusted the threshold and obtained a series of connectivity matrices with different sparsities. Using graph theory analysis, we characterized the global topological properties of brain functional networks using the parameters of smallworldness $(\sigma)$, which was defined as [25]

$$
\sigma=\frac{\gamma}{\lambda}=\frac{C_{p}^{\text {real }} / C_{p}^{\text {rand }}}{L_{p}^{\text {real }} / L_{p}^{\text {rand }}},
$$

TABLE 2: Regions of interest (ROI) defined in AAL template.

\begin{tabular}{|c|c|c|c|}
\hline \multirow{2}{*}{ Region name } & \multirow{2}{*}{ Abbr. } & \multicolumn{2}{|c|}{ Labels } \\
\hline & & $\mathrm{L}$ & $\mathrm{R}$ \\
\hline Precentral gyrus & PreCG & 1 & 2 \\
\hline Superior frontal gyrus, dorsolateral & SFGdor & 3 & 4 \\
\hline Superior frontal gyrus, orbital & SFGorb & 5 & 6 \\
\hline Middle frontal gyrus & MFG & 7 & 8 \\
\hline Middle frontal gyrus, orbital & MFGorb & 9 & 10 \\
\hline Inferior frontal gyrus, opercular & IFGoper & 11 & 12 \\
\hline Inferior frontal gyrus, triangular & IFGtri & 13 & 14 \\
\hline Inferior frontal gyrus, orbital & IFGorb & 15 & 16 \\
\hline Rolandic operculum & ROL & 17 & 18 \\
\hline Supplementary motor area & SMA & 19 & 20 \\
\hline Olfactory cortex & OLF & 21 & 22 \\
\hline Superior frontal gyrus, medial & SFGmed & 23 & 24 \\
\hline Superior frontal gyrus, medial orbital & SFGmorb & 25 & 26 \\
\hline Gyrus rectus & REG & 27 & 28 \\
\hline Insula & INS & 29 & 30 \\
\hline Anterior cingulate gyrus & ACG & 31 & 32 \\
\hline Median cingulate gyrus & MCG & 33 & 34 \\
\hline Posterior cingulate gyrus & PCG & 35 & 36 \\
\hline Hippocampus & HIP & 37 & 38 \\
\hline Parahippocampal gyrus & PHIP & 39 & 40 \\
\hline Amygdala & AMYG & 41 & 42 \\
\hline Calcarine fissure & CAL & 43 & 44 \\
\hline Cuneus & CUN & 45 & 46 \\
\hline Lingual gyrus & LING & 47 & 48 \\
\hline Superior occipital gyrus & SOG & 49 & 50 \\
\hline Middle occipital gyrus & MOG & 51 & 52 \\
\hline Inferior occipital gyrus & IOG & 53 & 54 \\
\hline Fusiform gyrus & FG & 55 & 56 \\
\hline Postcentral gyrus & PoCG & 57 & 58 \\
\hline Superior parietal gyrus & SPG & 59 & 60 \\
\hline Inferior parietal gyrus & IPG & 61 & 62 \\
\hline Supramarginal gyrus & SMG & 63 & 64 \\
\hline Angular gyrus & ANG & 65 & 66 \\
\hline Precuneus & $\mathrm{PCNU}$ & 67 & 68 \\
\hline Paracentral lobule & PCL & 69 & 70 \\
\hline Caudate & CAU & 71 & 72 \\
\hline Putamen & PUT & 73 & 74 \\
\hline Pallidum & PAL & 75 & 76 \\
\hline Thalamus & THA & 77 & 78 \\
\hline Heschl gyrus & HES & 79 & 80 \\
\hline Superior temporal gyrus & STG & 81 & 82 \\
\hline Superior temporal gyrus, temporal pole & STGp & 83 & 84 \\
\hline Middle temporal gyrus & MTG & 85 & 86 \\
\hline Middle temporal gyrus, temporal pole & MTGp & 87 & 88 \\
\hline Inferior temporal gyrus & ITG & 89 & 90 \\
\hline
\end{tabular}

where $\gamma$ and $\lambda$ are normalized clustering coefficient and normalized path length, respectively. The normalized clustering coefficient is the ratio of clustering coefficient of a real 
network $C_{p}^{\text {real }}$ to that of a random network $C_{p}^{\text {rand }}$, and the normalized path length is defined as the ratio of characteristic path length of a real network $L_{p}^{\text {real }}$ to that of a random network $L_{p}^{\text {rand }}$.

Here, clustering coefficient $C_{p}$ is a measure of the degree to which nodes in a network cluster together and is defined as

$$
C_{p}=\frac{1}{n} \sum_{i=1}^{n} \frac{2 E_{i}}{D_{\text {nod }}(i)\left(D_{\text {nod }}(i)-1\right)},
$$

where $D_{\text {nod }}(i)$ is the degree of the node $i$, which is defined in the following part of this section, $E_{i}$ is the total number of edges connecting the node $i$ with the nodes which are the nearest neighbors to the node $i$, and $N$ is the number of nodes in the network.

Characteristic path length $L_{p}$ is the average shortest path length between two nodes over all pairs of nodes and is defined as

$$
L_{p}=\frac{\sum_{i} \sum_{j} L_{i j}}{n(n-1)},
$$

where $n$ is the number of nodes in the network and $L_{i j}$ is the shortest path length between nodes $i$ and $j$.

A network is said to have the property of small-worldness if it satisfies $\lambda \approx 1$ and $\gamma \gg 1$ or $\sigma=\gamma / \lambda \gg 1$.

It has been demonstrated that the sparsity of $12 \%$ is close to an optimal threshold at which most informative network edges are retained and disconnections between nodes are rare $[26,27]$. Therefore, we chose $12 \%$ as the sparsity threshold for analysis of nodal topological properties, including betweenness centrality, nodal degree, and nodal efficiency, which are defined as follows.

(1) Betweenness Centrality. Betweenness centrality $C_{b}$ is a measure of the number of times that a node is along the shortest path between other two nodes, reflecting the importance of the individual node in the overall network structure. It is computed as follows:

$$
C_{b}=\sum_{s \neq i \neq t \in N} \frac{\delta_{s t}(i)}{\delta_{s t}}
$$

where $N$ is the set of all nodes in the network, $\delta_{s t}$ is the total number of shortest paths from node $s$ to node $t$, and $\delta_{s t}(i)$ is the number of the paths that pass through the node $i$.

(2) Nodal Degree. Nodal degree of the node $i\left(D_{\text {nod }}(i)\right)$ in a network is the number of connections linking the node with all other nodes and is defined as

$$
D_{\text {nod }}(i)=\sum_{j \in N} a_{i j}
$$

where $N$ is the set of all nodes in the network and $a_{i j}$ is the connection status between node $i$ and node $j\left(a_{i j}=1\right.$ when the connection exists, and $a_{i j}=0$ otherwise).
(3) Nodal Efficiency. Nodal efficiency of the node $i\left(E_{\text {nod }}(i)\right)$ is the mean of the inverse shortest path length from node $i$ to all other nodes and is defined as

$$
E_{\text {nod }}(i)=\frac{1}{n} \sum_{j \neq i} \frac{1}{L_{i j}}
$$

where $n$ is the number of nodes in the network and $L_{i j}$ is the shortest path length between nodes $i$ and $j$.

\subsection{Statistical Analysis}

2.6.1. Analysis of Whole-Brain Functional Connectivity. When constructing whole-brain network using resting-state fMRI, the correlation matrix characterized the brain functional connectivity pattern for each subject. To identify significant connectivity pattern in each group, one-sample $t$-test was performed to find significantly positive correlation in deaf group and normal controls, respectively, indicating significant connections between pairs of regions throughout the whole brain. To compare differences of connectivity patterns between groups, Fisher's $R$-to- $Z$ transformation was first performed and then two-sample $t$-test was conducted on the correlation matrices between prelingually deaf adolescents and normal controls. These statistical results were corrected for multiple comparisons using partial Bonferroni correction which considers the correlation between the dependent variables. This correction method was implemented using the Simple Interactive Statistical Analysis Bonferroni tool (http://www.quantitativeskills.com/sisa/calculations/bonfer.htm), which optimally balanced Type-I and Type-II errors. When performing one-sample $t$-test, the mean correlation coefficient between all variables in the matrix was 0.2236 in control group and 0.2274 in deaf group, leading to an equivalent corrected $p$ of $7.94 e-5$ for control group and $8.63 e-5$ for deaf group (number of tests $=90 *$ $89 / 2=4005$ ), respectively. For analysis of group differences, the mean correlation coefficient between pairs of variables for all subjects was 0.1624 , resulting in an equivalent corrected $p$ of $4.83 e-5$.

2.6.2. Analysis of Network Topological Properties. To present the properties of brain functional network for each group, the property of small-worldness was analyzed at different sparsities (from $10 \%$ to $45 \%$ ) for prelingually deaf adolescents and normal controls, respectively. Then, two-sample $t$-test was performed between two groups to figure out the group differences.

As described above, we chose $12 \%$ as the sparsity threshold for analysis of nodal topological properties. First, the mean betweenness centrality, mean nodal degree, and mean nodal efficiency were calculated for each node in deaf and normal groups, respectively. Then, we ranked them among all the nodes with the highest score (90) for the highest betweenness centrality, nodal degree, and nodal efficiency, respectively, and the scores for each measure were summed up for each node. The top $20 \%$ nodes (18 out of 90 nodes) with high scores were identified as hub nodes in each group. The three nodal topological parameters were then compared 


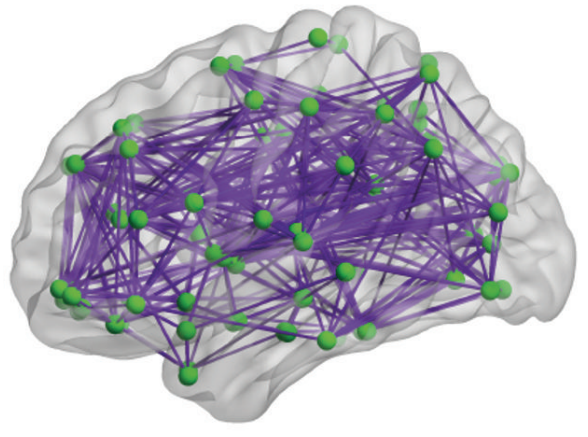

Control

(a)

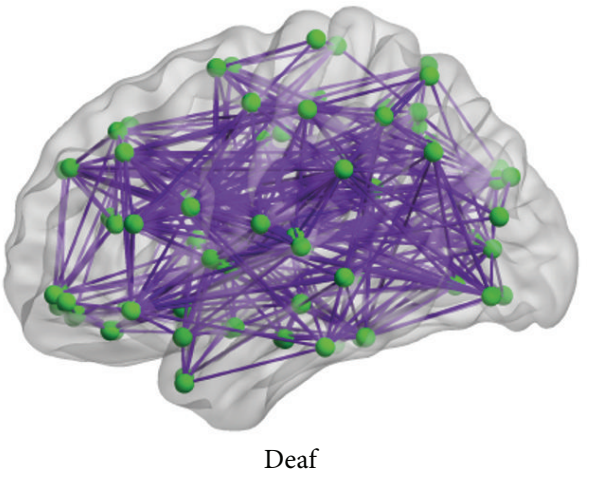

(b)

FIGURE 1: 3D views of functional connectivity patterns in normal controls (a) and prelingually deaf adolescents (b).

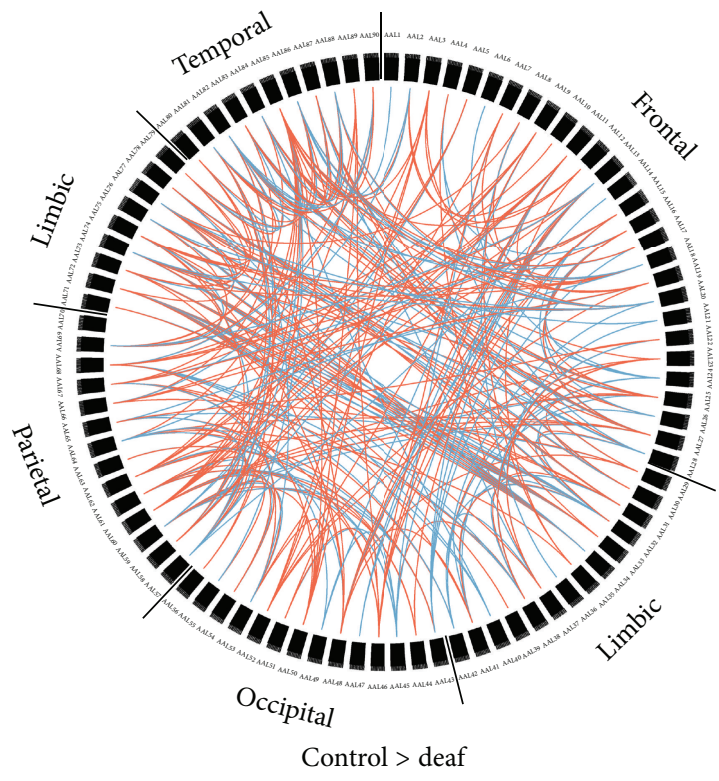

(a)

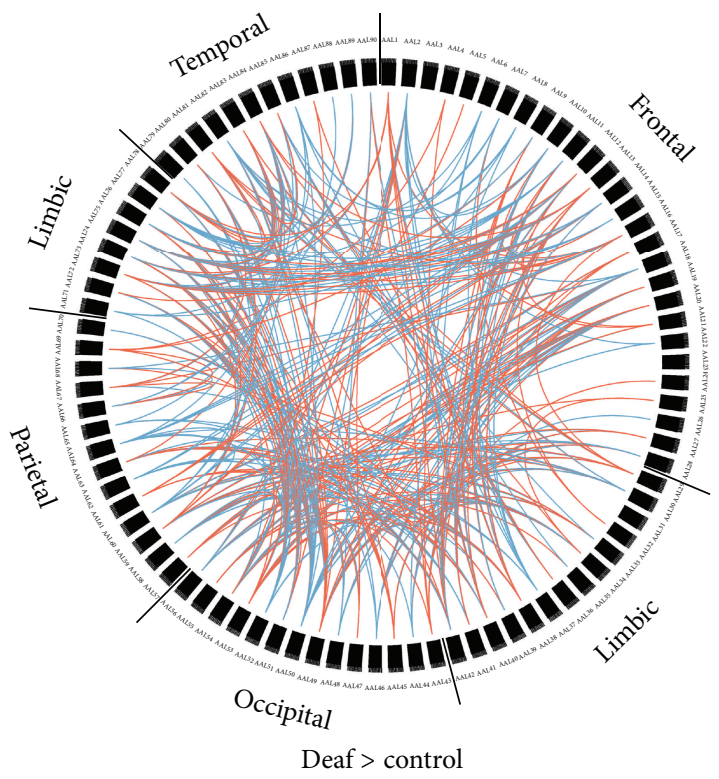

(b)

FIGURE 2: Group differences of brain functional connectivity between prelingually deaf adolescents and normal controls (uncorrected, $p<$ 0.05). (a) represents stronger functional connectivity in normal controls, while (b) shows stronger connectivity in prelingually deaf subjects. The red color indicates unilateral connections, and the blue color indicates bilateral connections.

between two groups for the hubs using two-sample $t$-test and the results were corrected for multiple comparisons using partial Bonferroni correction as well.

\section{Results}

3.1. Whole-Brain Functional Connectivity Pattern. Using onesample $t$-test, significant functional connectivity was detected for both prelingually deaf adolescents and normal controls after partial Bonferroni correction (as shown in Figure 1). The $3 \mathrm{D}$ view in Figure 1 was visualized with the BrainNet Viewer (http://www.nitrc.org/projects/bnv) [28]. The figure shows functional connectivity was widely distributed throughout the whole brain in both groups.

To identify group differences of functional connectivity throughout the whole brain, connectivity matrices were compared between two groups. Figure 2 shows the group differences before correction for multiple comparisons. From the figure, we can see that prelingually deaf adolescents show weaker connectivity between the regions within the temporal lobe, but stronger connectivity between regions in the occipital lobe and regions in the limbic system and temporal lobe. After partial Bonferroni correction, prelingually deaf adolescents showed significantly increased functional connectivity between the right superior gyrus (SPG) and right insula (INS) and between the left middle temporal gyrus (MTG) and right posterior cingulate gyrus (PCG) when compared with normal controls (see Figure 3 ). Besides, significantly decreased connectivity was found in deaf group between the right SPG and left middle frontal gyrus (orbital part, MFGorb) and between the right postcentral gyrus (PoCG) and left inferior frontal gyrus (opercular part, IFGoper). 


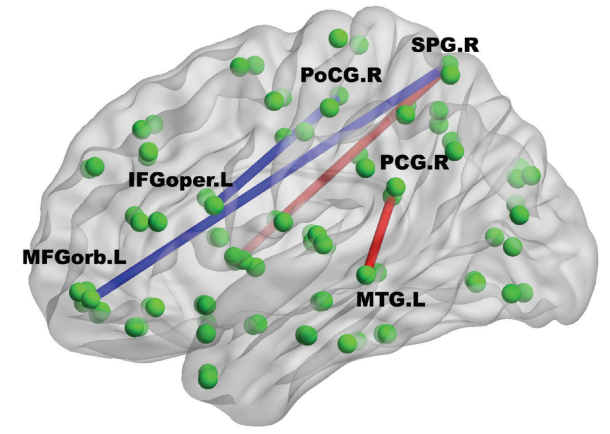

Figure 3: The 3D view for group differences after correction for multiple comparisons. While the warm color represents stronger connectivity in prelingually deaf adolescents compared to normal controls, the cold color suggests weaker connectivity.

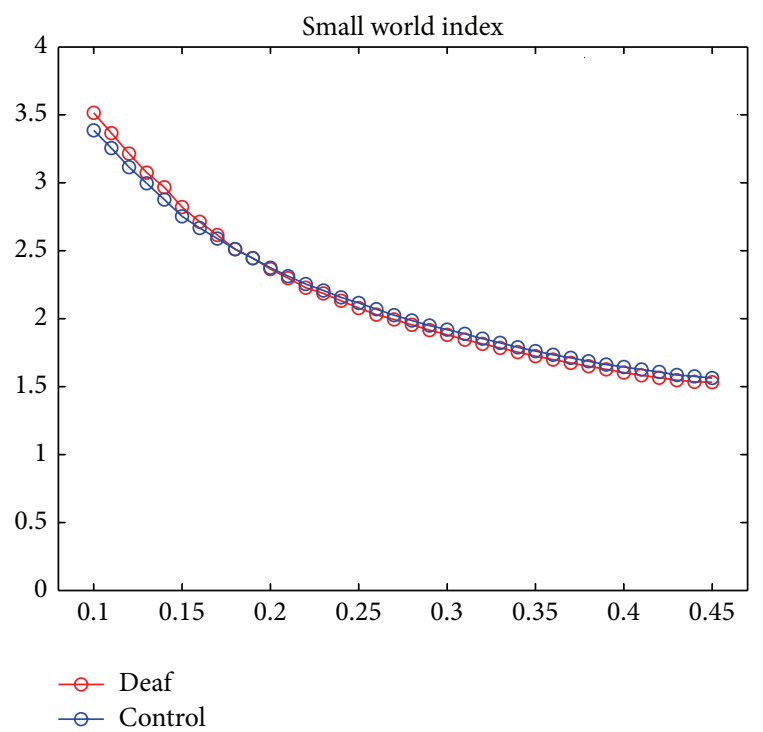

FIgURE 4: Small world index $\sigma$ across sparsities from $10 \%$ to $45 \%$ with an interval of $1 \%$ in prelingually deaf adolescents (red color) and normal controls (blue color). Both of the two groups present the property of small-worldness since $\sigma \gg 1$.

3.2. Properties of Brain Functional Network. Small-worldness $\sigma$ of brain functional network was measured at different sparsity (from $10 \%$ to $45 \%$ with an interval of $1 \%$ ) in deaf group and control group, respectively. As shown in Figure 4, we found $\sigma \gg 1$ across all the sparsities in both groups, indicating that the network has the property of smallworldness. We did not find significant differences of the small world index between prelingually deaf adolescents and normal controls.

Besides the global network property, the regional topological properties, including betweenness centrality, nodal degree, and nodal efficiency, were calculated in each ROI. Figure 5 shows the mean values for these regional properties in control and deaf groups.

3.3. Hubs of Brain Network. The hub in the network is supposed to have relatively high betweenness centrality, nodal degree, and nodal efficiency. Based on the method described in Section 2, we identified 18 regions out of 90 ROIs as the hubs in each group at the sparsity of $12 \%$ since a network with this sparsity tends to retain the edges with most information and ensure disconnection is rare. As shown in Figure 6 and Table 3, ten brain regions, including the right rolandic operculum (ROL), left gyrus rectus (REG), bilateral calcarine fissure (CAL), right lingual gyrus (LING), left putamen (PUT), right pallidum (PAL), bilateral Heschl gyrus (HES), and right superior temporal gyrus (STG), were the hubs in both deaf and control groups. Besides, the middle and superior frontal gyri and cuneus (CUN) were hubs in deaf group but not in control group, while the right precentral gyrus (PreCG), bilateral hippocampus (HIPP), and supramarginal gyrus (SMG) were hubs only in control group.

3.4. Group Differences of Topological Parameters for Hubs. There were a total of 26 brain regions identified as hubs either in deaf or in control groups. The nodal topological parameters, including betweenness centrality, nodal degree, and nodal efficiency, were compared for these nodes between prelingually deaf adolescents and normal controls. Significant differences did not survive after correction for multiple comparisons. However, we found trends for group differences, as listed in Table 3. Betweenness centrality is higher in the left superior frontal gyrus (orbital part, SFGorb) $(p=0.0204$, not corrected) and is lower in the left ROL ( $p=0.0480)$, SMG $(p=0.0251)$, and PAL $(p=0.0221)$ in deaf group relative to control group. Besides, prelingually deaf adolescents have higher nodal degree in the left middle frontal gyrus (orbital part, MFGorb) $(p=0.0484)$ and left superior frontal gyrus (medial part, SFGmed) $(p=0.0302)$ and lower nodal efficiency in the left ROL ( $p=0.0367)$, left hippocampus $(p=0.0442)$, and left pallidum $(p=0.0015)$.

\section{Discussions}

The present study employed resting-state fMRI and graph theory analysis to investigate changes of brain functional network in prelingually deaf adolescents. Functional connectivity was widely distributed in both deaf and control groups, and significant differences were found for connections between the right SPG and right insula, the left MTG and right PCG, the right SPG and left MFGorb, and the right PoCG and left IFGoper. The whole-brain network has the property of small-worldness in both prelingually deaf adolescents and normal controls. Ten regions were identified as the hubs in both groups. While the middle and superior frontal gyri and CUN were the hubs that emerged only in deaf group, the right PreCG, bilateral HIPP, and SMG were hubs only in control group. These findings provide new evidence for brain reorganization in prelingually deaf adolescents and could help to understand the working mechanism in the period of brain development after hearing loss.

In prelingually deaf adolescents, functional connectivity was widely distributed as observed in normal controls but differed in the connectivity pattern. Significantly increased 


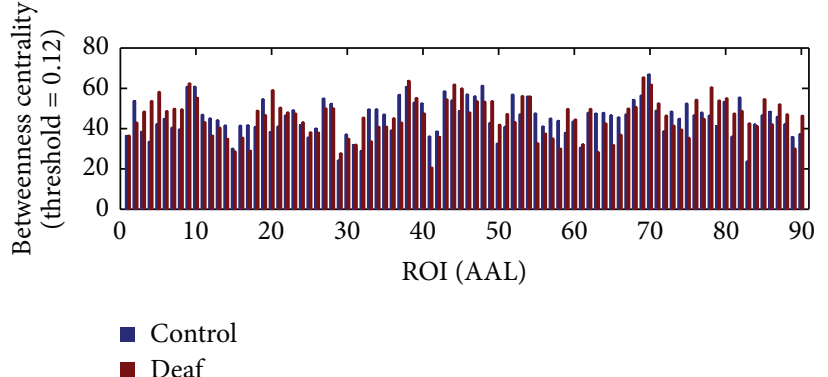

(a)

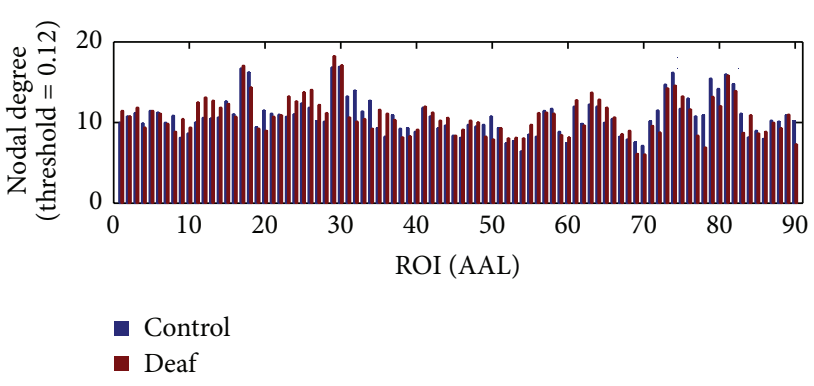

(b)

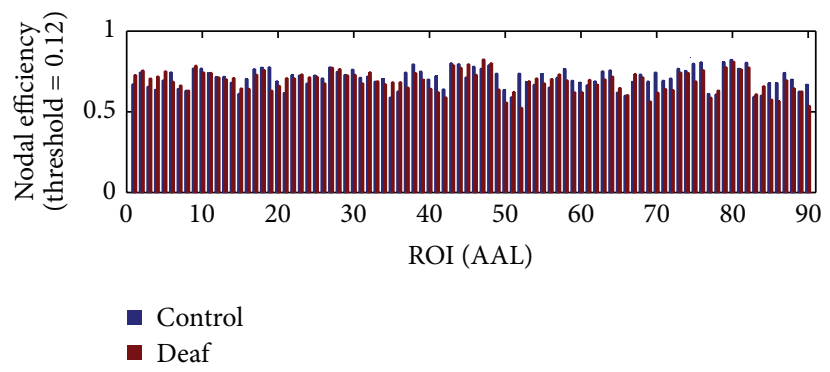

(c)

FIgURE 5: Bar plots for the average value for regional topological properties of 90 ROIs, including betweenness centrality (a), nodal degree (b), and nodal efficiency (c), in control and deaf groups. The blue color indicates the control group, and the red color represents the deaf group.

$\mathrm{L}$

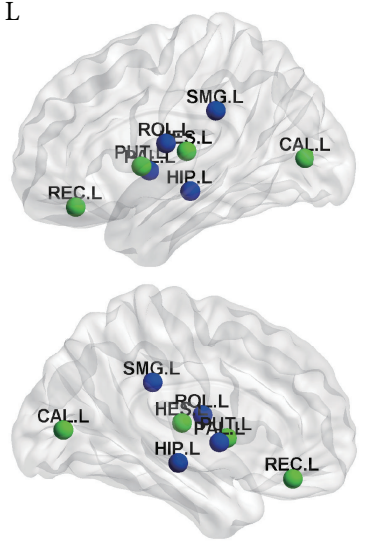

Control

(a)

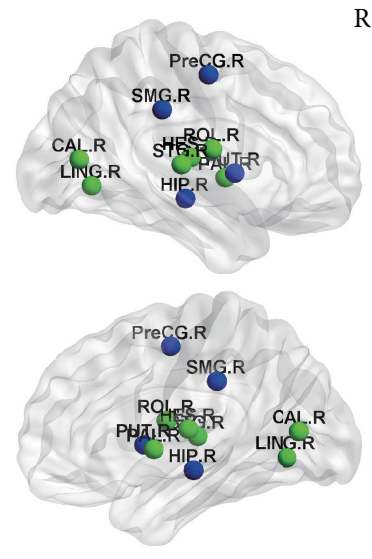

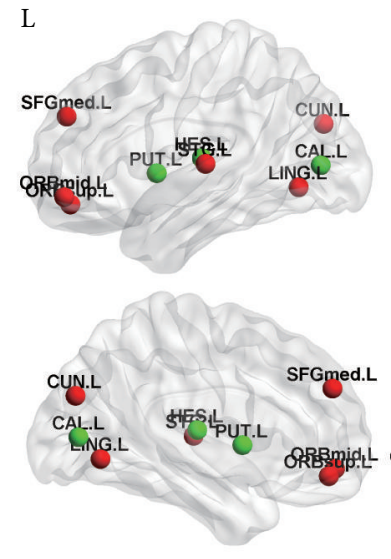

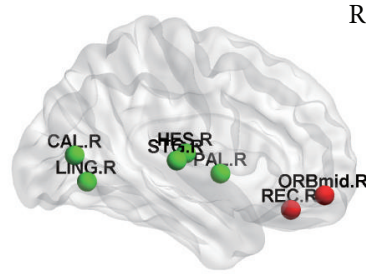

(b)

FIGURE 6: Hubs for brain functional networks in control (a) and deaf (b) groups. The hubs identified in both groups are shown in green color. While the hubs for the control group only are present with blue color, the hubs for the deaf group only are shown in red color.

connectivity was detected between the right SPG and right insula and between the left MTG and right PCG. The insula and PCG are in limbic system, which are thought to play an important role in recruiting relevant brain regions for sensory information processing [29]. The SPG is supposed to receive considerable visual input and is involved in visualspatial relations [30]. Besides, the MTG has been reported to be one of the critical nodes in the brain's language network, which can access word meaning while reading [31]. Therefore, our findings of increased connectivity between these regions suggest that the limbic system in prelingually deaf adolescents could reinforce the processing of the visual and verbal information due to the deprivation of auditory sensory processing. Additionally, we found decreased functional connectivity between the right SPG and left MFGorb and between the right PoCG and left IFGoper. Evidence suggests that the left MFG is the specific brain region for Chinese reading [32], and the left IFG is extremely important for language 
TABLE 3: Group differences of nodal topological parameters in hubs (not corrected).

\begin{tabular}{|c|c|c|c|c|c|c|c|c|}
\hline \multirow{2}{*}{ Brain region } & \multicolumn{2}{|c|}{ Hubs } & \multicolumn{2}{|c|}{$\mathrm{BC}$} & \multicolumn{2}{|c|}{ ND } & \multicolumn{2}{|c|}{$\mathrm{NE}$} \\
\hline & Deaf & Control & $\mathrm{D}>\mathrm{C}$ & $\mathrm{D}<\mathrm{C}$ & $\mathrm{D}>\mathrm{C}$ & $\mathrm{D}<\mathrm{C}$ & $\mathrm{D}>\mathrm{C}$ & $\mathrm{D}<\mathrm{C}$ \\
\hline PreCG.R & nonhub & hub & ns & ns & ns & ns & ns & Ns \\
\hline SFGorb.L & hub & nonhub & 0.0204 & ns & ns & ns & ns & Ns \\
\hline MFGorb.L & hub & nonhub & ns & ns & 0.0484 & ns & ns & Ns \\
\hline MFGorb.R & hub & nonhub & ns & ns & ns & ns & ns & Ns \\
\hline ROL.L & nonhub & hub & ns & 0.0480 & ns & ns & ns & 0.0367 \\
\hline ROL.R & hub & hub & ns & ns & ns & ns & ns & Ns \\
\hline SFGmed.L & hub & nonhub & ns & ns & 0.0302 & ns & ns & Ns \\
\hline REG.L & hub & hub & ns & ns & ns & ns & ns & Ns \\
\hline REG.R & hub & nonhub & ns & ns & ns & ns & ns & Ns \\
\hline HIP.L & nonhub & hub & ns & ns & ns & ns & ns & 0.0442 \\
\hline HIP.R & nonhub & hub & ns & ns & ns & ns & ns & Ns \\
\hline CAL.L & hub & hub & ns & ns & ns & ns & ns & Ns \\
\hline CAL.R & hub & hub & ns & ns & ns & ns & ns & Ns \\
\hline LING.L & hub & nonhub & ns & ns & ns & ns & ns & Ns \\
\hline LING.R & hub & hub & ns & ns & ns & ns & ns & Ns \\
\hline SMG.L & nonhub & hub & ns & 0.0251 & ns & ns & ns & Ns \\
\hline SMG.R & nonhub & hub & ns & ns & ns & ns & ns & Ns \\
\hline CUN.L & hub & nonhub & ns & ns & ns & ns & ns & Ns \\
\hline PUT.L & hub & hub & ns & ns & ns & ns & ns & Ns \\
\hline PUT.R & nonhub & hub & ns & ns & ns & ns & ns & Ns \\
\hline PAL.L & nonhub & hub & ns & 0.0221 & ns & ns & ns & 0.0015 \\
\hline PAL.R & hub & hub & ns & ns & ns & ns & ns & Ns \\
\hline HES.L & hub & hub & ns & ns & ns & ns & ns & Ns \\
\hline HES.R & hub & hub & ns & ns & ns & ns & ns & Ns \\
\hline STG.L & hub & nonhub & ns & ns & ns & Ns & ns & Ns \\
\hline STG.R & hub & hub & ns & ns & ns & Ns & ns & Ns \\
\hline
\end{tabular}

D > C: deaf $>$ control; D < C: deaf < control; ns: not significant.

comprehension and production [33]. The decreased connectivity might be caused by the inferior skills of reading or speaking observed in most of the deaf subjects $[34,35]$.

Although it is not significant, we found a trend of significance for group differences in functional connectivity pattern. Connectivity between the regions within the temporal lobe was found to be weaker in prelingually deaf adolescents, indicating that the auditory cortex are less activated to auditory stimuli. In contrast, stronger connectivity was observed between regions in the occipital lobe and regions in the temporal lobe, which were identified as primary visual and auditory cortices, respectively. This result is consistent with one of our findings in the previous study [23], which detected significantly stronger structural connections between visual and auditory systems in prelingually deaf adolescents.

Using graph theory analysis, whole-brain network holds the property of small-worldness in both prelingually deaf adolescents and normal controls. The small world index was almost the same across all the sparsities from $10 \%$ to $45 \%$ (with an interval of $1 \%$ ) between the two groups (see Figure 3). This result suggests that the effectiveness of information transferring of the brain network was not affected even without auditory inputs in prelingually deaf adolescents.
In the brain network, hubs are regions playing an important role in facilitating communication among parallel, distributed brain networks [36]. Eighteen hubs were identified in each group. In control group, the hubs included the right PreCG, bilateral ROL, left REG, bilateral HIPP, bilateral CAL, right LING, bilateral PUT, bilateral PAL, bilateral HES, and right STG. Compared with healthy adolescents, middle and superior frontal gyri, left LING, left CUN, left STG, and right REG were hubs only observed in prelingually deaf adolescents. Evidences show that the STG is the primary auditory cortex and is involved in phonological processing for speech perception and production as well [37,38]. Although not activated by auditory stimuli, auditory regions could be activated by other stimuli [1-4] as stated in Section 1 . Therefore, auditory regions might become a brain hub connecting with other brain regions. In the hubs of deaf subjects, the LING and CUN are associated with visual processing $[39,40]$. To adapt to deficient auditory input, prelingually deaf adolescents could rely critically on vision to interact with the external environment. Our findings of the hubs in LING and CUN suggest the improvement of visual performance in deaf adolescents, supporting cross-modal changes in visual processing. Of note, deaf subjects recruited in the present study learn Chinese Sign Language (CSL). It has 
been demonstrated that the middle and superior frontal gyri can be activated during observing and imitating CSL [41]. We speculated that the hubs of MFG and SFG only observed in prelingually deaf subjects might be caused by the use of CSL. All the above changes indicate brain reorganizations after hearing loss in prelingually deaf adolescents.

There are still some limitations in the present study. First, the sample size is relatively small. We recruited 16 prelingually deaf adolescents and 16 normal controls, and 15 subjects remained for each group after removing subjects with excessive head movement. More subjects will be scanned in further studies. Second, the effects of clinical parameters, such as the information for the use of sign language and hearing aids, were not analyzed in the present study, which will be taken into consideration in future.

\section{Conclusions}

The present study employed resting-state fMRI and graph theory to investigate alterations of brain functional network in prelingually deaf adolescents compared to normal controls. Functional connectivity was widely distributed in prelingually deaf adolescents. Increased connectivity was significantly found between the limbic system and visual and language-related regions, suggesting reinforcement of the processing for the visual and verbal information in deaf adolescents. Decreased connectivity was detected between the visual regions and language regions possibly due to inferior reading or speaking skills in deaf subjects. Graph theory analysis revealed that brain functional network holds small-worldness in both prelingually deaf adolescents and normal controls. Compared with healthy adolescents, middle and superior frontal gyri, left LING, left CUN, left STG, and right REG were hubs only observed in prelingually deaf adolescents. These hubs were involved in visual, language, and auditory processing, reflecting brain reorganization to adapt to deficient auditory input.

\section{Conflict of Interests}

The authors declare that there is no conflict of interests regarding the publication of this paper.

\section{Authors' Contribution}

Wenjing Li and Jianhong Li contributed equally to this paper.

\section{Acknowledgments}

This work is supported by 863 Project (2013AA013803), the National Natural Science Foundation of China (NSFC) (no. 81271557, no. 61271151, and no. 61228103), Youth Innovation Promotion Association CAS, the Beijing Postdoctoral Research Foundation (no. 2014ZZ-8), the Project Funded by China Postdoctoral Science Foundation (no. 2015M570911), and the Basic Research Foundation Project of Beijing University of Technology. The authors thank all the subjects and their families for the time and effort they dedicate to the authors' research.

\section{References}

[1] M. MacSweeney, R. Campbell, G. A. Calvert et al., "Dispersed activation in the left temporal cortex for speech-reading in congenitally deaf people," Proceedings of the Royal Society B: Biological Sciences, vol. 268, no. 1466, pp. 451-457, 2001.

[2] H. Yoshida, Y. Kanda, H. Takahashi, I. Miyamoto, and K. Chiba, "Observation of cortical activity during speech stimulation in prelingually deafened adults with cochlear implantation by positron emission tomography-computed tomography," Annals of Otology, Rhinology and Laryngology, vol. 120, no. 8, pp. 499504, 2011.

[3] I. Fine, E. M. Finney, G. M. Boynton, and K. R. Dobkins, "Comparing the effects of auditory deprivation and sign language within the auditory and visual cortex," Journal of Cognitive Neuroscience, vol. 17, no. 10, pp. 1621-1637, 2005.

[4] R. S. Dewey and D. E. Hartley, "Cortical cross-modal plasticity following deafness measured using functional near-infrared spectroscopy," Hearing Research, vol. 325, pp. 55-63, 2015.

[5] K. Emmorey, J. S. Allen, J. Bruss, N. Schenker, and H. Damasio, "A morphometric analysis of auditory brain regions in congenitally deaf adults," Proceedings of the National Academy of Sciences of the United States of America, vol. 100, no. 17, pp. 10049-10054, 2003.

[6] D. K. Shibata, "Differences in brain structure in deaf persons on MR imaging studied with voxel-based morphometry," American Journal of Neuroradiology, vol. 28, no. 2, pp. 243-249, 2007.

[7] J. Li, W. Li, J. Xian et al., "Cortical thickness analysis and optimized voxel-based morphometry in children and adolescents with prelingually profound sensorineural hearing loss," Brain Research, vol. 1430, pp. 35-42, 2012.

[8] W. Li, J. Li, J. Xian et al., "Alterations of grey matter asymmetries in adolescents with prelingual deafness: a combined VBM and cortical thickness analysis," Restorative Neurology and Neuroscience, vol. 31, no. 1, pp. 1-17, 2013.

[9] O. Sporns, G. Tononi, and R. Kötter, “The human connectome: a structural description of the human brain," PLoS Computational Biology, vol. 1, no. 4, article e42, 2005.

[10] O. Sporns, "From simple graphs to the connectome: networks in neuroimaging," NeuroImage, vol. 62, no. 2, pp. 881-886, 2012.

[11] T. Nakamura, F. G. Hillary, and B. B. Biswal, "Resting network plasticity following brain injury," PLoS ONE, vol. 4, no. 12, Article ID e8220, 2009.

[12] E. Bullmore and O. Sporns, "Complex brain networks: graph theoretical analysis of structural and functional systems," Nature Reviews Neuroscience, vol. 10, no. 3, pp. 186-198, 2009.

[13] Y. He and A. Evans, "Graph theoretical modeling of brain connectivity," Current Opinion in Neurology, vol. 23, no. 4, pp. 341-350, 2010.

[14] D. S. Bassett and E. Bullmore, "Small-world brain networks," Neuroscientist, vol. 12, no. 6, pp. 512-523, 2006.

[15] J. Wang, L. Wang, Y. Zang et al., "Parcellation-dependent smallworld brain functional networks: a resting-state fmri study," Human Brain Mapping, vol. 30, no. 5, pp. 1511-1523, 2009.

[16] Y. Liu, M. Liang, Y. Zhou et al., "Disrupted small-world networks in schizophrenia," Brain, vol. 131, no. 4, pp. 945-961, 2008 . 
[17] K. Wu, Y. Taki, K. Sato, H. Qi, R. Kawashima, and H. Fukuda, "A longitudinal study of structural brain network changes with normal aging," Frontiers in Human Neuroscience, vol. 7, no. 3, article 113, 2013.

[18] Z. Liu, L. Ke, H. Liu, W. Huang, and Z. Hu, "Changes in topological organization of functional PET brain network with normal aging," PLoS ONE, vol. 9, no. 2, Article ID e88690, 2014.

[19] M. P. van den Heuvel, R. C. W. Mandl, C. J. Stam, R. S. Kahn, and H. E. Hulshoff Pol, "Aberrant frontal and temporal complex network structure in schizophrenia: a graph theoretical analysis," Journal of Neuroscience, vol. 30, no. 47, pp. 15915-15926, 2010.

[20] E. J. Sanz-Arigita, M. M. Schoonheim, J. S. Damoiseaux et al., "Loss of 'small-world' networks in Alzheimer's disease: graph analysis of fMRI resting-state funcitonal connectivity," PLoS ONE, vol. 5, no. 11, Article ID e13788, 2010.

[21] B. M. Tijms, A. M. Wink, W. de Haan et al., "Alzheimer's disease: connecting findings from graph theoretical studies of brain networks," Neurobiology of Aging, vol. 34, no. 8, pp. 2023-2036, 2013.

[22] E. Kim, H. Kang, H. Lee et al., "Morphological brain network assessed using graph theory and network filtration in deaf adults," Hearing Research, vol. 315, pp. 88-98, 2014.

[23] Y. Li, J. R. Booth, D. Peng et al., "Altered intra- and inter-regional synchronization of superior temporal cortex in deaf people," Cerebral Cortex, vol. 23, no. 8, pp. 1988-1996, 2013.

[24] W. Li, J. Li, Z. Wang et al., "Grey matter connectivity within and between auditory, language and visual systems in prelingually deaf adolescents," Restorative Neurology and Neuroscience, vol. 33, no. 3, pp. 279-290, 2015.

[25] D. J. Watts and S. H. Strogatz, "Collective dynamics of 'smallworld' networks,” Nature, vol. 393, no. 6684, pp. 440-442, 1998.

[26] A. Lord, D. Horn, M. Breakspear, and M. Walter, "Changes in community structure of resting state functional connectivity in unipolar depression," PLoS ONE, vol. 7, no. 8, Article ID e41282, 2012.

[27] M. Rubinov, S. A. Knock, C. J. Stam et al., "Small-world properties of nonlinear brain activity in schizophrenia," Human Brain Mapping, vol. 30, no. 2, pp. 403-416, 2009.

[28] M. Xia, J. Wang, and Y. He, "BrainNet viewer: a network visualization tool for human brain connectomics," PLOS ONE, vol. 8, no. 7, Article ID e68910, 2013.

[29] T. P. White, V. Joseph, S. T. Francis, and P. F. Liddle, "Aberrant salience network (bilateral insula and anterior cingulate cortex) connectivity during information processing in schizophrenia," Schizophrenia Research, vol. 123, no. 2-3, pp. 105-115, 2010.

[30] M. Corbetta, G. L. Shulman, F. M. Miezin, and S. E. Petersen, "Superior parietal cortex activation during spatial attention shifts and visual feature conjunction," Science, vol. 270, no. 5237, pp. 802-805, 1995.

[31] D. J. Acheson and P. Hagoort, "Stimulating the brain's language network: syntactic ambiguity resolution after TMS to the inferior frontal gyrus and middle temporal gyrus," Journal of Cognitive Neuroscience, vol. 25, no. 10, pp. 1664-1677, 2013.

[32] L. H. Tan, H.-L. Liu, C. A. Perfetti, J. A. Spinks, P. T. Fox, and J.-H. Gao, "The neural system underlying Chinese logograph reading," NeuroImage, vol. 13, no. 5, pp. 836-846, 2001.

[33] S. G. Costafreda, C. H. Y. Fu, L. Lee, B. Everitt, M. J. Brammer, and A.S. David, "A systematic review and quantitative appraisal of fMRI studies of verbal fluency: role of the left inferior frontal gyrus," Human Brain Mapping, vol. 27, no. 10, pp. 799-810, 2006.
[34] I. Parasnis, V. J. Samar, and G. P. Berent, "Deaf adults without attention deficit hyperactivity disorder display reduced perceptual sensitivity and elevated impulsivity on the Test of Variables of Attention (T.O.V.A.)," Journal of Speech, Language, and Hearing Research, vol. 46, no. 5, pp. 1166-1183, 2003.

[35] R. Hollingsworth, A. K. Ludlow, A. Wilkins, R. Calver, and P. M. Allen, "Visual performance and ocular abnormalities in deaf children and young adults: a literature review," Acta Ophthalmologica, vol. 92, no. 4, pp. 305-310, 2014.

[36] K. Hwang, M. N. Hallquist, and B. Luna, "The development of hub architecture in the human functional brain network," Cerebral Cortex, vol. 23, no. 10, pp. 2380-2393, 2013.

[37] M. A. Howard, I. O. Volkov, R. Mirsky et al., "Auditory cortex on the human posterior superior temporal gyrus," Journal of Comparative Neurology, vol. 416, no. 1, pp. 79-92, 1999.

[38] B. R. Buchsbaum, G. Hickok, and C. Humphries, "Role of left posterior superior temporal gyrus in phonological processing for speech perception and production," Cognitive Science, vol. 25, no. 5, pp. 663-678, 2001.

[39] A. Mechelli, G. W. Humphreys, K. Mayall, A. Olson, and C. J. Price, "Differential effects of word length and visual contrast in the fusiform and lingual gyri during reading," Proceedings of the Royal Society B: Biological Sciences, vol. 267, no. 1455, pp. 19091913, 2000.

[40] S. Vanni, T. Tanskanen, M. Seppä, K. Uutela, and R. Hari, "Coinciding early activation of the human primary visual cortex and anteromedial cuneus," Proceedings of the National Academy of Sciences of the United States of America, vol. 98, no. 5, pp. 2776-2780, 2001.

[41] Q. Li, S. Xia, and F. Zhao, "An FMRI picture study of Chinese sign language in functional cortex of prelingual deaf signers use computer," Communications in Computer and Information Science, vol. 158, pp. 348-355, 2011. 

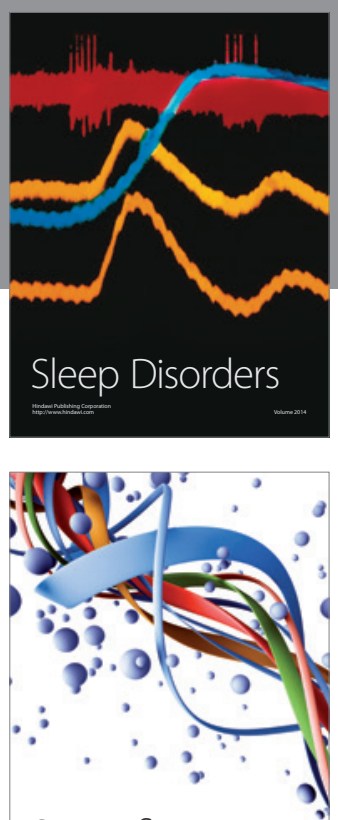

Scientifica
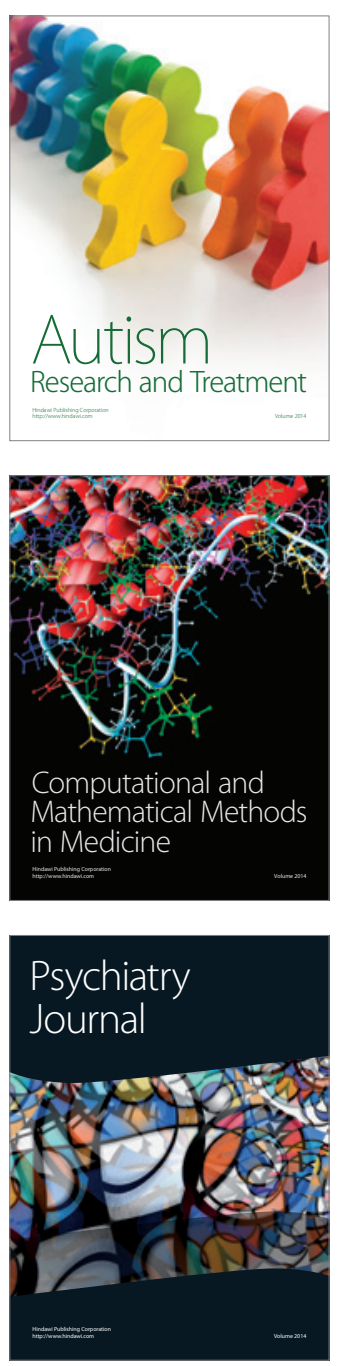
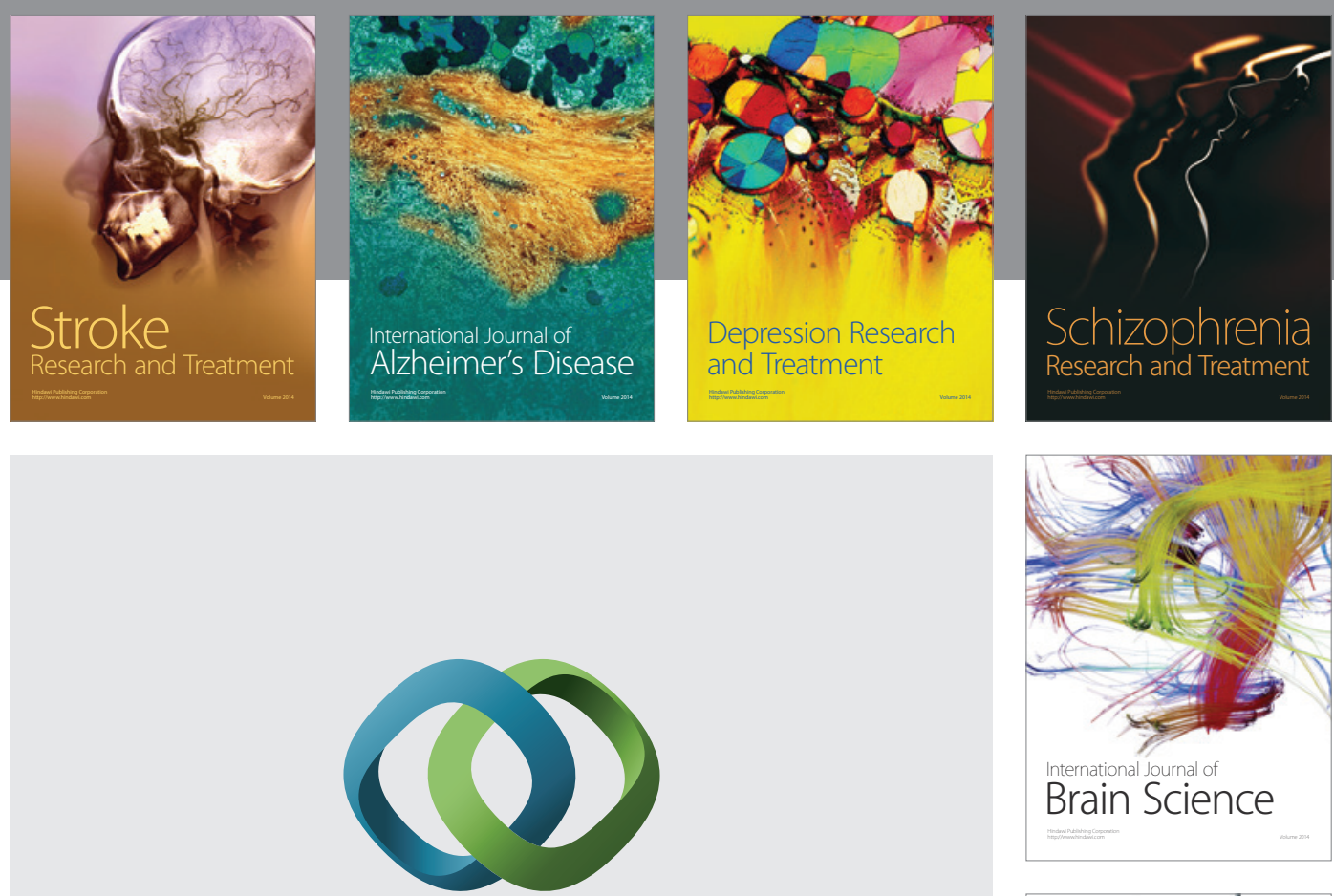

\section{Hindawi}

Submit your manuscripts at

http://www.hindawi.com
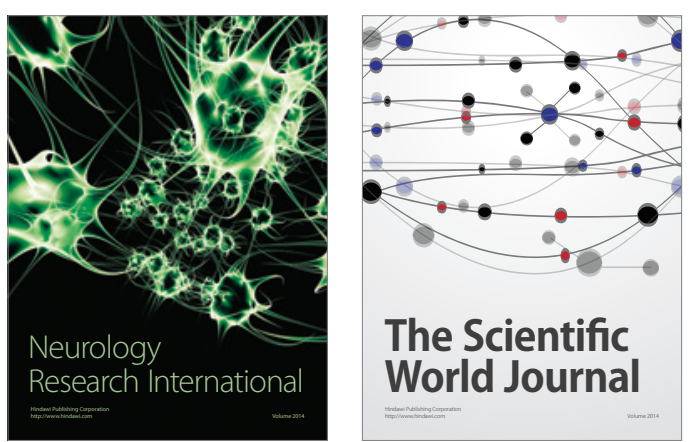

The Scientific World Journal

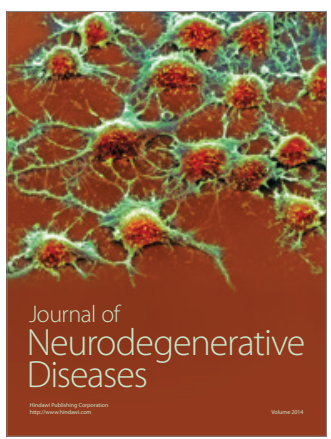

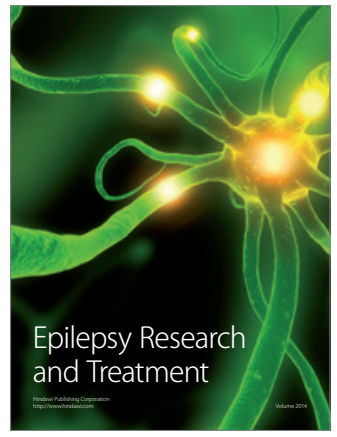

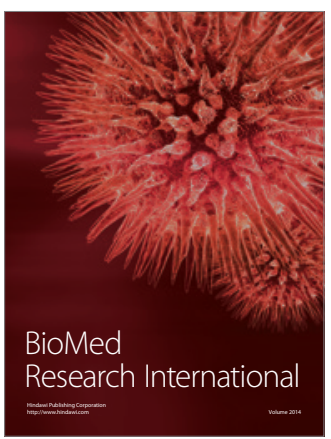

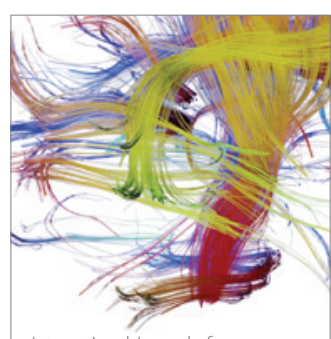

Brain Science

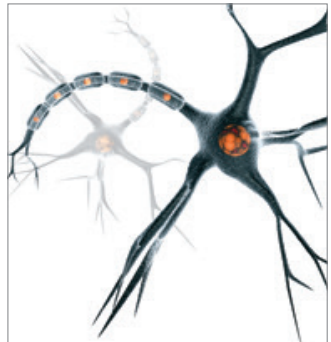

Neural Plasticity
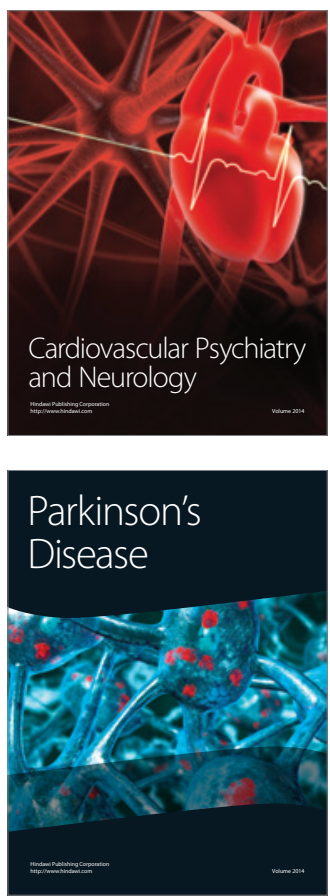